\title{
Partner or Perish: VA Health Services and the Emerging Bi-Directional Paradigm
}

\author{
Amy M. Kilbourne, $\mathrm{PhD}, \mathrm{MPH} \mathrm{H}^{1,2}$ and David Atkins, $\mathrm{MD}, \mathrm{MPH}^{1}$ \\ 'VA Health Services Research and Development Service, Department of Veterans Affairs, Washington, DC, USA; ${ }^{2}$ Department of Psychiatry, \\ University of Michigan, Ann Arbor, MI, USA.
}

J Gen Intern Med 29(Suppl 4):S817-9

DOI: $10.1007 / \mathrm{s} 11606-014-3050-3$

(c) Society of General Internal Medicine 2014

$\mathrm{T}$ he Veterans Health Administration (VHA) is the nation's largest integrated healthcare system and has the benefit of a sizeable budget for research to improve Veterans' health and healthcare. Thus, many outsiders assume that research has always been tightly integrated with VHA clinical operations and policy. In reality, for years researchers and operations leaders inhabited their separate silos, responding to the different priorities and time lines of their respective jobs. While connections between researchers and policy or clinical offices were numerous, with researchers serving as expert consultants in many initiatives, there were few incentives for the two groups to work closely together in a sustained effort. Policymakers wanted research that could help inform decisions, yet often found it lacked important context to make it relevant; however, policymakers didn't have time or capacity to help design more useful research. Similarly, while researchers wanted to see their work affect VHA care, the crucial time needed to directly work with partners to maximize the impact of their work in day-to-day practice and the important insights gained through this process were not rewarded in the same way as time spent writing grants or journal manuscripts. The reality in VA and in similar settings involving academically affiliated healthcare organizations is that partnership is hard, takes time and energy, can occasionally be frustrating or fruitless, and has to meet the needs of both partners to truly succeed.

VHA's Health Services Research and Development Service (HSR\&D) and the Quality Enhancement Research Initiative (QUERI) have made major efforts to enhance partnered research. Our aims in doing so are similar to those summarized in this issue's editorial by Selby and Slutsky - the more relevant the research is, the more likely it is to have an impact. ${ }^{1}$ Twelve of the articles in this issue describe partnered research at various stages, from conceptualizing partnered research to examples of findings borne from bi-directional collaborations with investigators and leaders from clinical operations. These articles cover a wide range of topics highly relevant to VHA policy and practice, including performance measure

Published online October 30, 2014 implementation on provider motivation, opioid management, suicide prevention, homelessness, medical home models, and communication of adverse events.

Partnered research has been defined as: "activities where researchers and practitioners work together, with different roles, to use research both to solve practical problems and to contribute to science." ${ }^{2}$ In HSR\&D and QUERI, successful research-operations partnerships tend to have three key ingredients: practical innovation, capacity through shared resources, and palpable impact across multiple levels. First, they involve innovation around solving practical clinical or implementation issues faced by frontline providers, clinical managers, and leaders through active communication and shared agenda between investigators and national program office(s) that lead to new perspectives and strategies. The VA Women's Health Practice-based Research Network is a prime example of a research-operational partner that led to several new projects focused on understudied health issues among women Veterans (i.e., reproductive health, cardiovascular disease). ${ }^{3}$ Second, successful partnered research involves shared resources (e.g., data, provider networks, personnel) that enable regional or national roll-out studies and flexibility for investigators to work on the policy-relevant issues. Examples of current initiatives involving shared data or provider networks include the VHA's medical home models ${ }^{4,5}$ and the suicide predictive analytic projects. ${ }^{6,7}$ In addition, optimal partnerships achieve clinical or policy impacts, ideally felt from the national or regional levels, through new policies as well as at the frontline provider levels through care improvement. The VA Homeless Health Services Research Initiative involved the VA's National Center on Homelessness among Veterans and clinical program offices in the active shaping and development of new clinical practices and policies focused on preventing and ending homelessness, notably through a critical review of the Housing First program, ${ }^{8}$ implementation of a medical home model for homeless Veterans, ${ }^{9}$ and dissemination of an outreach program for Veterans at risk of homelessness. ${ }^{10}$

Both HSR\&D and QUERI have promoted partnered research in complementary but distinct ways. Examples of HSR \&D-partnered research initiatives include the Collaborative Research to Enhance and Advance Transformation and Excellence (CREATE) ${ }^{11}$ and Centers of Innovation $(\mathrm{COIN})^{12}$ initiatives. HSR\&D takes a longer-term 
perspective to build the science that operations can use across initiatives to help predict what they need 35 years from now. In contrast, QUERI, whose goal is to help providers implement evidence-based practices using quality improvement methods, often employs a shorter time frame of 1-3 years.

Each of the ten QUERI Centers uses a strategic planning process that is shaped by investigators and clinical operational partners in order to implement projects that are more focused on implementation and contributing to best implementation practices. Some of the QUERI-based partnerships highlighted in this issue include strategies to facilitate provider implementation of primary care/mental health integration ${ }^{13}$ and VA acute stroke care centers. ${ }^{14}$ QUERI has also recently initiated several Partnered Evaluation Centers focused on new ways to promote patient-centered care, e-health specialty care services, staffing models for nursing, and reimbursement policies for caregivers.

How can we better support researchers in developing active, bi-directional partnerships with clinical operations? Many of the articles in this issue consist of the active ingredients of successful research-operations partnerships, outlined below:

Get Involved Early. Investigators need avenues to get involved early with the clinical and policy decisionmaking process; foremost by articulating the value added in having a researcher involved in new initiatives. By planning ahead and getting connected with operational partners, researchers can get involved earlier in the process to conduct rigorous studies that control for historical trends by using systematic designs that allow for the comparison of interventions or staggered dissemination of intervention resources more fairly and efficiently. HSR\&D and QUERI leaders are facilitating meetings where clinical offices can discuss their priorities and initiatives with interested researchers to help align research with the directions of the health system.

Value-Added. Researchers need to make it clear to partners what they are getting from research, such as expertise, methods, data access, etc. Many operational partners are reluctant to involve researchers because the research cycle is slow. In response, the VA has adopted policies for speeding the review process by more clearly defining when studies fall under human subjects research or quality improvement. ${ }^{15}$ For their part, researchers need to allow flexibility in developing studies with operational partners, in many cases forgoing lengthy assessments in favor of more practice data ascertainment. For example, it might be helpful for researchers to form a contractual relationship through a memorandum of understanding with clinical partners with more quick deliverables to garner their trust and facilitate a working relationship.

Mutually Beneficial. The relationship needs to be truly mutual-benefitting both parties. Ken Wells and Loretta Jones ${ }^{16}$ describe these types of partnerships as a process of "engagement," in the same way one envisions the term used to describe marriage. It takes trust, commitment, communication, and patience to continue the partnership over the long term. For example, researchers and partners work on a mutually agreeable protocol for reviewing study findings that allows the researcher to be independent while not blindsiding the operational partner. Researchers should also acknowledge the contribution of partners and allow partners to take credit for findings as a means to increase impact of the research. QUERI has recently adopted two measures of impact that appeal to partners and expand beyond papers and grants: whether the research findings have been communicated to key decision-makers, and whether the findings shaped or directly resulted in a change to clinical practice or policy.

Seek Input from Different Stakeholders. Researchers need to involve their end-users in the development and operationalization of research so that it stands a greater chance of being accepted and adopted in the real world. Some of the best ideas leading to new treatment discoveries and implementation methods come from end users. For health services research, the Patient Centers Outcomes Research Initiative (PCORI) program has led the way in involving consumers and patients in the funding decision process and has advocated that studies use a patient council or involve patients in the study team. For QUERI, the main end-users are frontline providers adopting effective programs, so greater efforts are needed to involve clinicians in the conceptual development and design of implementation studies. VA's Mental Health QUERI recently launched a Stakeholder Council consisting of frontline providers and Veteran representatives who review project ideas and make recommendations regarding the Center's strategic direction. The HMO Research Network is also developing patient engagement toolkits to facilitate more rapid implementation of patient-centered research and even help investigators recruit, train and support patient co-investigators. ${ }^{17}$

Overall, successful research-clinical partnerships in which both parties have a shared agenda and ongoing interaction can lead to increased probability that the research findings will be used in routine practice. Researchers and partners should strike an appropriate balance between rigor and time-sensitive opportunities in order to make more dramatic progress on implementing effective programs into clinical practice. While researchers should be flexible to partners' needs, they need to be in a position to challenge assumptions made by the partner, and the partner needs to be open to potential negative findings regarding their programs. The sweet spot might be achieved through the greater use of practical clinical trials, 
quality improvement mechanisms, and/or natural experiments. ${ }^{18}$ Ultimately, creating an environment of psychological safety between researchers and operational partners is crucial to maintaining a trusting relationship.

There is additional work to be done by funders, academic departments, and healthcare organizations if we are to truly move to a culture of partnership. Academic research settings, healthcare organizations, and funders need to create the right incentives to reward the hard work of partnership, both in promotion decisions for researchers and in the expectations of clinical leaders, so we can attract and support the best people and ideas. Funders also need to create clear expectations of when and what type of partnership is expected.

The current challenges VHA and other healthcare systems face in improving access, quality, and efficiency of care may provide unexpected opportunities for investigators to lead collaborative work. Researchers should strive to more actively promote alliances with program partners, and to ensure that frontline providers are actively involved in the development and implementation of new research initiatives to insure uptake and impact.

Corresponding Author: Amy M. Kilbourne, PhD, MPH; VA Health Services Research and Development Service, Department of Veterans Affairs, 810 Vermont Avenue NW, Washington, DC 20420, USA (e-mail: Amy.Kilbourne@va.gov).

\section{REFERENCES}

1. Selby JV, Slutsky JR. Practicing Partnered Research. J Gen Intern Med. doi:10.1007/s11606-014-3046-Z

2. Ovretveit J, Hempel S, Magnabosco JL, Mittman BS, Rubenstein LV, Ganz DA. Guidance for research-practice partnerships (R-PPs) and collaborative research. J Health Organ Manag. 2014;28(1):115-126.

3. Frayne SM, Carney DV, Bean-Mayberry B, et al. The VA women's health practice-based research network: amplifying women veterans' voices in VA Research. J Gen Intern Med. 2013;28(Suppl 2):504-509.
4. Nelson KM, Helfrich C, Sun H, Hebert PL, Liu CF, Dolan E, Taylor L, Wong E, Maynard C, Hernandez SE, Sanders W, Randall I, Curtis I, Schectman G, Stark R, Fihn SD. Implementation of the patient-centered medical home in the veterans health administration: associations with patient satisfaction, quality of care, staff burnout, and hospital and emergency department use. JAMA Intern Med. 2014;174(8):1350-1358. doi:10.1001/jamainternmed.2014.2488.

5. Zulman DM, Ezeji-Okoye SC, Shaw JG, et al. Partnered research in healthcare delivery redesign for high-need, high-cost patients: Development and Feasibility of an Intensive Management Patient Aligned Care Team (ImPACT). J Gen Intern Med. doi: 10.1007/s11606-014-3022-7

6. Bossarte RM, Knox KL, Piegari R, Altieri J, Kemp J, Katz IR. Prevalence and characteristics of suicide ideation and attempts among active military and veteran participants in a national health survey. Am J Public Health. 2012;102(Suppl 1):S38-S40. doi:10.2105/ AJPH.2011.300487.

7. Dobscha SK, Denneson LM, Kovas AE, et al. Correlates of suicide among veterans treated in primary care: Case-control study of a nationally representative sample. J Gen Intern Med. doi:10.1007/s11606-014-3028-1

8. Austin EL, Pollio DE, Holmes S, Schumacher J, White B, Lukas CV, Kertesz S. VA's expansion of supportive housing: successes and challenges on the path toward Housing First. Psychiatr Serv. 2014;65(5):641647. doi:10.1176/appi.ps.201300073

9. O'Toole TP, Bourgault C, Johnson EE, Redihan SG, Borgia M, Aiello R, Kane V. New to care: demands on a health system when homeless veterans are enrolled in a medical home model. Am J Public Health. 2013;103(Suppl 2):S374-S379. doi:10.2105/AJPH.2013.301632. Epub 2013 Oct 22.

10. Kilbourne AM, Abraham KM, Goodrich DE, Bowersox NW, Almirall D, Lai Z, Nord KM. Cluster randomized adaptive implementation trial comparing a standard versus enhanced implementation intervention to improve uptake of an effective re-engagement program for patients with serious mental illness. Implement Sci. 2013;8:136. doi:10.1186/17485908-8-136.

11. HSR\&D Collaborative Research to Enhance and Advance Transformation and Excellence (CREATE) Initiative; Available at: http:// www.hsrd.research.va.gov/centers/create/

12. VHA Centers of Innovation (COIN): available at: http:// www.hsrd.research.va.gov/centers/default.cfm

13. Kirchner JE, Ritchie MJ, Pitcock JA, Parker LE, Curran GM, Fortney JC. Outcomes of a partnered facilitation strategy to implement primary care - mental health. J Gen Intern Med. doi: 10.1007/s11606-014-3027-2

14. Damush TM, Miller KK, Plue L, et al. National implementation of acute stroke care centers in the Veterans Health Administration (VHA): Formative evaluation of the field's response. J Gen Intern Med. doi:10.1007/s11606014-3036-1

15. Department of Veterans Affairs. VHA Handbook 1058.05. VHA Operations Activities that May Constitute Research. October 28, 2011. Available at: http://www.va.gov/vhapublications/ViewPublication.asp?pub_ID=2456

16. Wells $\mathbf{K}$, Jones $\mathbf{L}$. The Promise of Community-Partnered Participatory Research: http://www.pcori.org/blog/the-promise-of-communitypartnered-participatory-research/

17. Pronovost $\mathbf{P}$, Jha AK. Did hospital engagement networks actually improve care? N Engl J Med. 2014;371(8):691-693. doi:10.1056/NEJMp1405800. 